\title{
Impact of Mismatching for Minor Histocompatibility Antigens on the Occurrence of Graft-Versus-Host Disease
}

\author{
E. Goulmy ${ }^{1}$, E. Blokland ${ }^{1}$, J. W. Gratama ${ }^{1}$, F. E.Zwaan ${ }^{2}$, J. M.J.J.Vossen ${ }^{3}$, B. Speck ${ }^{4}$, and J.J.van Rood ${ }^{1}$ \\ ${ }^{1}$ Department of Immunohaematology and Blood Bank, University Hospital, Leiden, The Netherlands \\ ${ }^{2}$ Isolation Pavilion, University Hospital, Leiden, The Netherlands \\ ${ }^{3}$ Department of Pediatrics, University Hospital, Leiden, The Netherlands \\ ${ }^{4}$ Kantonsspital Basel, Switzerland
}

The results in human bone marrow transplantation are still not fuily satisfactory. Despite the selection of HLA identical siblings as bone marrow donors for patients with severe aplastic anemia or hematologic mallgnancles, Graft-versus-host Disease (GvHD) occurs in approximately 20-70\% of the patients depending on their age. This compltcation can be caused by disparity for the products of minor Histocorpatibility (minor H) systens.

Previously, we reported on the presence of cytotoxic T lymphocytes (CTLs) in a patient suffering from severe chron $1 \mathrm{c}$ GuHD. Those CTLs, which we re

demonstrated fron two months after bone marrow grafting onwards, were directed against patient's own pre-transplant lymphocytes. Analysis of the in vitro cytotoxic activity of the patient's post-transplant 1 ymphocytes demonstrated the presence of a minor $H$ antigen of which the recognition was HLA restricted (Goulmy et al. 1982).

Thls observation prompted us to continue our search for mfnor $H$ antigens and their role in bone marrow transplantation. We investigated post-transplant 1 ymphocytes from a series $(n=19)$ of recipients of HLA fdentical bone marrow grafts for the presence of anti-host cytotoxic activity. Such CTL activity could be detected post bone marrow transplant in five patients suffering from GuHD, but was absent in patients without GuHD (for review see: Goulmy 1985). Further analysis of the cytotoxic activity patterns of the post-transplant lyothocytes of the latter five patients, revealed five different CTL populations each directed against different minor H antigens. Four of the $f i v e$ minor $H$ antignes were recognized in an HLA restricted fashion (Goulmy 1985). Expansion of the five CTL populations provided us with large amounts of cellular typing reagents specific for these minor $H$ antigens.

In order to obtaln information about the relevance of minor $H$ antigens in the pathogenesis of $G \mathrm{vHD}$, we performed an retrospective typing analysis for these five different minor $H$ antigens, on a series of HLA identical bone narrow donor/recipient corbinations $(n=47)$.
The results of this analysis (see table) denonstrate that incompatibilities for one (or more) minor $\mathrm{H}$ antigens between HLA identical donor and recipient were found in the group of patients suffering from GvHD. Consequently, when more than one bone marrow donor is available, cellular typing for minor $H$ antigens may be helpful in avoiding one of the risk factors for GvHD.

Table. Cellular typing of human minor $\mathrm{H}$ antigens with minor $H$ antigen speclfic CTLs.

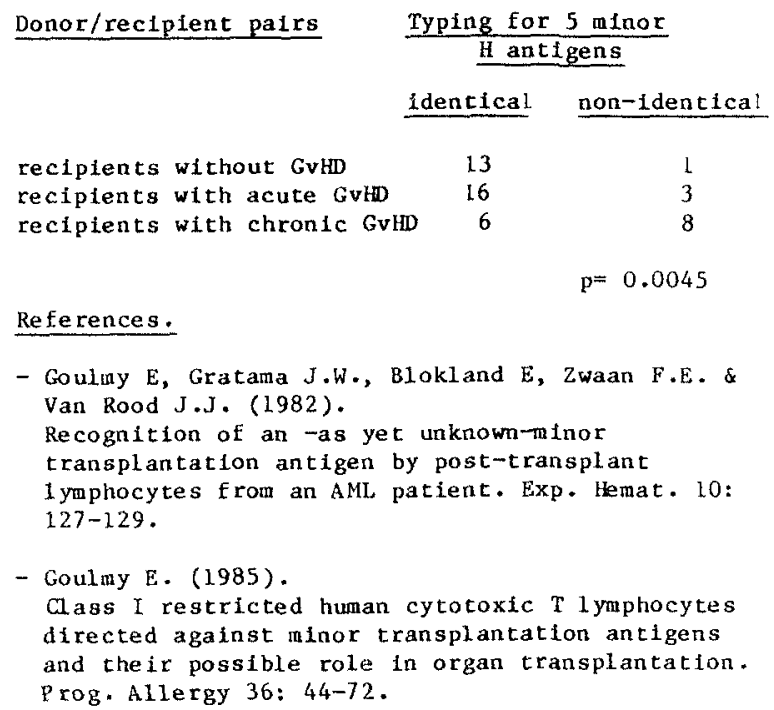
16 6

References.

- Gouliny E, Gratama J.W., Blokland E, Zwaan F.E. \& Van Rood J.J. (1982).

Recognition of an -as yet unknown-minor transplantation antigen by post-transplant 1 ynphocytes from an AML patient. Exp. Hemat. 10: $127-129$.

- Goulmy E. (1985).

Class I restricted human cytotoxic $T$ lymphocytes directed against minor transplantation antigens and their possible role in organ transplantation. prog. Allergy 36: 44-72.

Supported in part by the Dutch Foundation for Medfcal Research (FUnGo) which is subsidized by the Dutch Organization for the Advancement of Pure Research ( 2 WO), the $J \cdot A$. Cohen Instltute for Radiopathology and Radiation Protection (IRS). 